\title{
Electric-field control of spin current generation and detection in ferromagnet-free $\mathrm{SrTiO}_{3}$-based nanodevices
}

Felix Trier ${ }^{1}$, Diogo C. Vaz ${ }^{1}$, Pierre Bruneel ${ }^{3}$, Paul Noël ${ }^{2}$, Albert Fert ${ }^{1}$, Laurent Vila ${ }^{2}$, JeanPhilippe Attané ${ }^{2}$, Agnès Barthélémy ${ }^{1}$, Marc Gabay ${ }^{3}$, Henri Jaffrès ${ }^{1}$ and Manuel Bibes ${ }^{1 *}$

${ }^{1}$ Unité Mixte de Physique, CNRS, Thales, Univ. Paris-Sud, Université Paris-Saclay, 91767, Palaiseau, France

${ }^{2}$ Univ. Grenoble Alpes, CEA, CNRS, Spintec, 38000 Grenoble, France

${ }^{3}$ LPS, Univ. Paris-Sud, Université Paris-Saclay, 91767, Palaiseau, France

*e-mail: manuel.bibes@cnrs-thales.fr

Keywords: Spin current generation/detection, Spin Hall effect, $\mathrm{SrTiO}_{3}$, two-dimensional electron gas, nanodevices

Abstract: Spintronics entails the generation, transport, manipulation and detection of spin currents, usually in hybrid architectures comprising interfaces whose impact on performance is detrimental. In addition, how spins are generated and detected is generally material specific and determined by the electronic structure. Here, we demonstrate spin current generation, transport and electrical detection, all within a single non-magnetic material system: a $\mathrm{SrTiO}_{3}$ two-dimensional electron gas (2DEG) with Rashba spin-orbit coupling. We show that the spin current is generated from a charge current by the 2D spin Hall effect, transported through a channel and reconverted into a charge current by the inverse 2D spin Hall effect. Furthermore, by adjusting the Fermi energy with a gate voltage we tune the generated and detected spin polarization and relate it to the complex multiorbital band structure of the 2DEG. We discuss the leading mechanisms of the spin-charge interconversion processes and argue for the potential of quantum oxide materials for future all-electrical low-power spin-based logic. 
The two-dimensional electron gas (2DEG) that forms at the surface ${ }^{1}$ of $\mathrm{SrTiO}_{3}$ (STO) or at its interface with oxides ${ }^{2,3}$ or metals ${ }^{4}$ possesses several fascinating properties such as high electron mobility ${ }^{5}$ and 2D superconductivity ${ }^{6}$. Importantly, in this 2DEG inversion symmetry is broken, which allows for the existence of Rashba spin-orbit coupling (SOC) ${ }^{7}$. Another remarkable feature of STO $2 \mathrm{DEGs}$ is the strong tunability of their carrier density and related electronic properties with a gate voltage ${ }^{8}$. In particular, the electric-field dependence of the Rashba $\mathrm{SOC}^{9}$ makes the STO 2DEG an attractive platform to design agile spin-orbitronics architectures.

Fig. 1a shows the Fermi surface of a prototypical Rashba 2DEG, with two Fermi contours along which the spin is locked perpendicularly to the momentum $k$, and having opposite spin chiralities. Firstly, when a charge current is applied along $x\left(j_{c}^{\text {inject }}\right)$, the Fermi contours are displaced by $\Delta k$ along $-x$, which results in the generation of a transverse spin density oriented along $y, \int s d y \neq 0$, through the direct Edelstein effect $^{10}(\mathrm{EE})$. This spin density can then diffuse (with a diffusion constant $D_{s}$ ) and produces a transverse spin current in the material or in adjacent layers. The inverse Edelstein effect ${ }^{11}$ (IEE) is the reciprocal of the direct EE: the injection of a spin density oriented along $y$ would cause opposite but inequivalent shifts of the Fermi contours along $x$, leading to the generation of a charge current.

In addition, after the displacement of Fermi contours by $\Delta k$, electrons see their spins precessing around the new expected spin direction, which tilts the spins along $+z$ for $k_{y}>0$ and $-z$ for $k_{y}<0$, thus creating a spin current polarized along $z$ in the $y$ direction $\left(j_{s}\right)$; this is the 2D spin Hall effect (2D-SHE) ${ }^{12}$. So far in STO 2DEGs, the $\mathrm{IEE}^{13}$ has been more intensely studied than the direct $\mathrm{EE}^{14}$ or the $2 \mathrm{D}-\mathrm{SHE}^{15}$, and has been shown to result in a very efficient spincharge conversion ${ }^{13,16}$. The figure of merit $\lambda_{\text {IEE }}$ was found to strongly depend in amplitude and sign on a gate voltage as the Fermi energy was varied through the peculiar electronic band structure of the $2 \mathrm{DEG}^{13,17}$, exhibiting a characteristic crossover in the sign of the effective Rashba SOC ${ }^{18}$. Those properties, together with the high conversion efficiency, offer interesting prospects for spin-orbitronics devices ${ }^{19,20}$. 
a

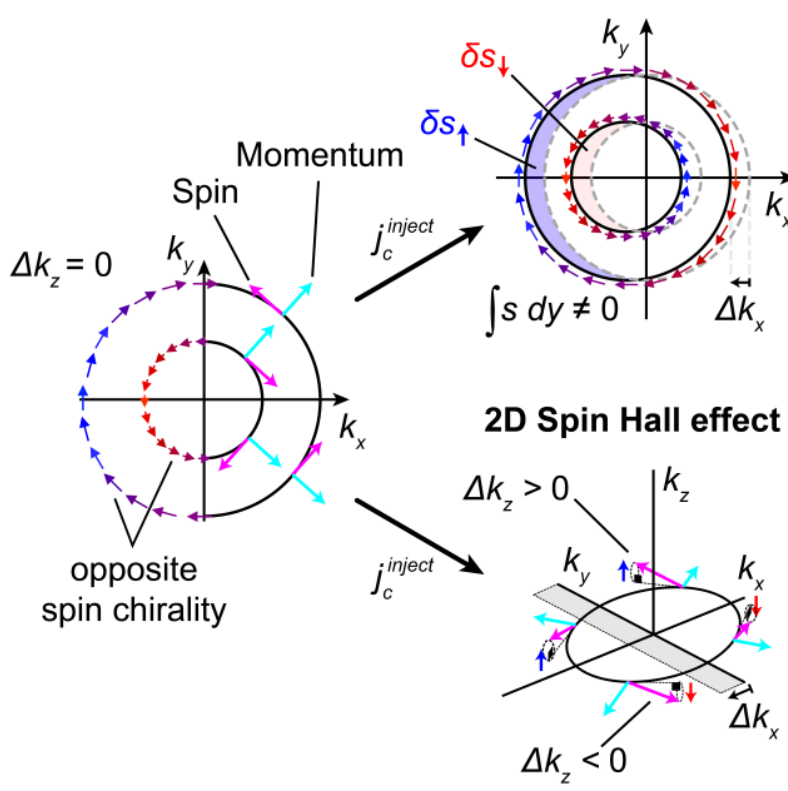

C

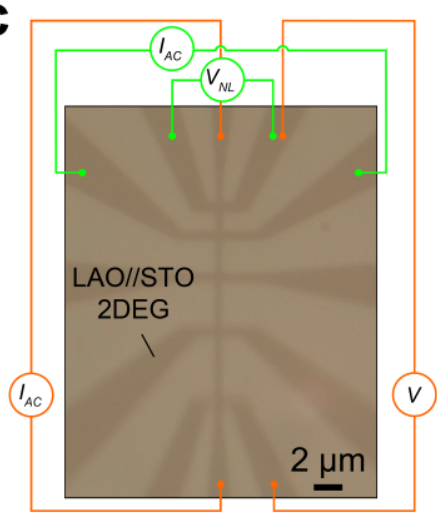

Edelstein effect b

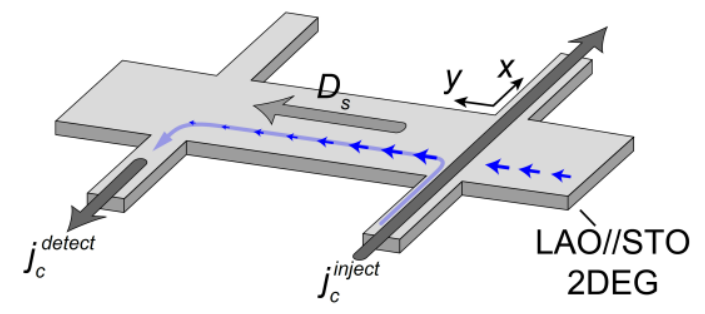

2D Spin Hall effect

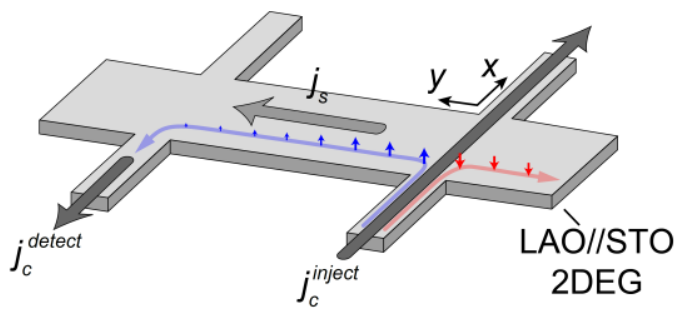

d

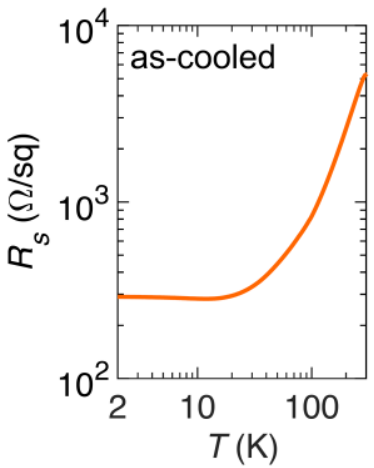

danle spin precession

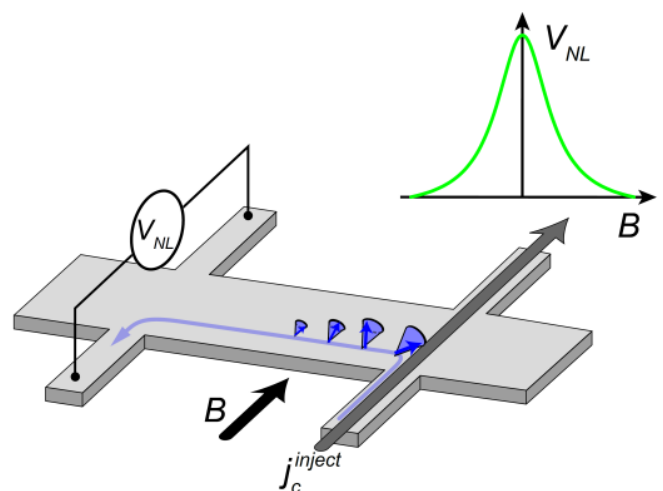

Figure 1. a, Because of the Rashba effect in $\mathrm{LaAlO}_{3} / / \mathrm{SrTiO}_{3}(\mathrm{LAO} / / \mathrm{STO})$, the spin degeneracy is lifted, leading to concentric Fermi contours with opposite spin chirality where the spin is locked perpendicular to the momentum. Through the direct Edelstein effect an injected charge current $\left(j_{c}^{\text {inject }}\right)$ along $x$ will generate a transverse spin accumulation along $y, \int s d y \neq 0$. Additionally, after displacing the Fermi contours by $\Delta k_{x}$, the spins will not be exactly perpendicular to $k$ and will thus start to precess around to local Rashba field to eventually align along the new spin direction. While precessing, the spins acquire a finite component along $+z$ for $k_{y}>0$ and $-z$ for $k_{y}<0$; this is the two-dimensional (2D) spin Hall effect, which ultimately results in a net spin polarization along z. $\boldsymbol{b}$, top panel, The spin accumulation generated by Edelstein effect will diffuse $\left(D_{s}\right)$ along $y$ in the Hall bar channel, before getting converted back into a detectable 
charge current at a neighbouring Hall probe $\left(j_{c}^{\text {detect }}\right)$ through the inverse Edelstein effect; bottom panel, the spin polarization along z caused by the 2D spin Hall effect will lead to a spin current in the $y$ direction $\left(j_{s}\right)$, reconverted into a charge current at a neighbouring Hall probe $\left(j_{c}^{\text {detect }}\right)$ through the inverse $2 D$ spin Hall effect. $c$, left panel, optical microscope image of a Hall bar device with a representative measurement configuration of non-local resistance (green) and local resistance (orange) indicated; right panel, sheet resistance, $R_{s}$, as a function of temperature $T$ for the LAO//STO 2DEG, consistent with its usual metallic behaviour. $d$, Applying a magnetic field $\underline{B}$ along $x$, perpendicularly to the spins diffusing in the channel, results in spin precession that progressively lowers the non-local voltage signal, $V_{N L}$ as the $B$ field is increased (Hanle effect).

Here we take advantage of the ability of STO 2DEGs to efficiently interconvert spin and charge currents, transport spins over long distances and strongly respond to carrier density modulation by electrostatic gating, in order to realize an agile monolithic planar spintronic device free from ferromagnets. LAO//STO Hall bar devices (see Fig. 1c) were fabricated by conventional electron-beam lithography and resist soft-mask lift-off ${ }^{21}$ (see Methods). The devices shows $n$-type metallic transport characteristics, comparable to those of unpatterned interfaces ${ }^{22}$, with the sheet resistance reducing from $5 \mathrm{k} \Omega / \mathrm{sq}$ to $300 \Omega / \mathrm{sq}$ as the temperature decreases from $300 \mathrm{~K}$ to $2 \mathrm{~K}$ (see Fig. 1c).

In the absence of ferromagnetic reference contacts, we probe the spin transport in the channel through Hanle experiments (see Fig. 1d). As sketched in Fig. 1b, the application of a charge current along $x$ in one arm of the Hall bar $\left(j_{c}^{\text {inject }}\right)$ generates a spin accumulation oriented along $y$ through the direct EE that diffuses sideways $\left(D_{s}\right)$ in the channel towards the second arm (Fig. 2b). The applied charge current can also generate a spin current polarized along $z$ through the 2D-SHE that similarly is transported towards the second arm $\left(j_{s}\right)$. At the second arm, the spin current is converted back into a charge current through the IEE or the inverse $2 \mathrm{D}-\mathrm{SHE}\left(j_{c}^{\text {detect }}\right)$, that we detect as a non-local voltage. Applying a magnetic field along $x$ (that is, perpendicularly to the spins diffusing in the channel) causes the spins to precess, and thus generates a Hanle effect ${ }^{23,24}$, as sketched in Fig. $1 d$. During this spin precession, the 
original spin polarization is lost, consequently lowering $j_{c}^{\text {detect }}$ and ultimately reducing the non-local voltage measured.

a

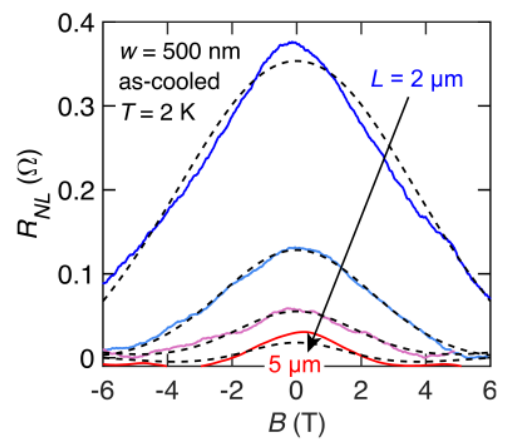

b

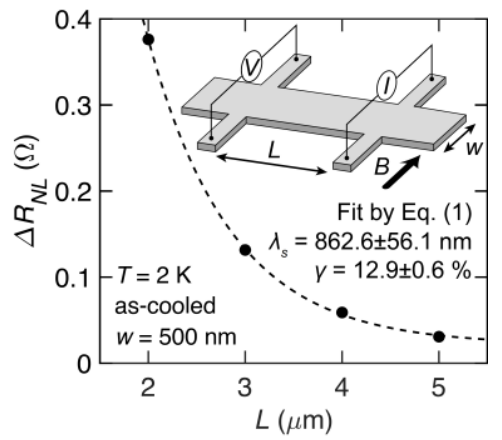

C

\begin{tabular}{cccc}
\multicolumn{4}{l}{ Fit by Eq. (2) } \\
\hline$L(\mu \mathrm{m})$ & \multicolumn{1}{l}{$\gamma(\%)$} & $\lambda_{s}(\mathrm{~nm})$ & \multicolumn{1}{c}{$\tau_{s}(\mathrm{ps})$} \\
\hline 2 & $12.3 \pm 0.6$ & $819 \pm 53$ & $2.14 \pm 0.23$ \\
3 & $12.3 \pm 0.3$ & $825 \pm 16$ & $2.02 \pm 0.06$ \\
4 & $12.3 \pm 0.9$ & $888 \pm 39$ & $2.05 \pm 0.13$ \\
5 & $13.6 \pm 8.2$ & $905 \pm 255$ & $2.03 \pm 0.79$ \\
\hline
\end{tabular}

Figure 2. $a$, Non-local resistance, $R_{N L}$ as a function of the magnetic field, $B$, for probe spacings between $2 \mu \mathrm{m}$ and $5 \mu \mathrm{m}$ at $2 \mathrm{~K}$. The fit of the data to Eqs. (1-2) is shown as dashed lines. $\boldsymbol{b}$, The non-local resistance amplitude, $\Delta R_{N L}$ as a function of probe spacing, L. c, Table of extracted fitting parameters from the fits in $\boldsymbol{a}$.

Fig. 2a displays the Hanle signals, $V_{N L} / j_{c}^{\text {inject }}=R_{N L}$, for various distances between the charge injection and detection probes, $L$ (see the measurement configuration in the inset of Fig. 2b). Herein, Hanle curves with a typical Lorentzian shape are obtained. Since the local sheet resistance contribution to the measured non-local resistance could give rise to spurious signals, we have designed the Hall bars to have a $L / w$ ratio always greater than 4 such that with $R_{N L}=R_{s}{ }^{*} \exp (-\pi L / w)$ the local contribution to the nonlocal voltage is negligible (less than $0.001 \%)$. Therefore by studying the amplitude $\Delta R_{N L}$ of the signal as a function of the probe spacing, we conclude that this non-local signal originates from the voltage conversion of a generated transverse spin-signal, expected to reduce exponentially with $L$ in the diffusive transport regime due to the increased spin dephasing time by ${ }^{23}$ :

$$
\Delta R_{N L}(L)=\frac{w}{2 \lambda_{s}} \gamma^{2} R_{s} e^{-\frac{L}{\lambda_{s}}}
$$


where $w$ is the Hall bar channel width, $\lambda_{s}$ is the spin diffusion length and $\gamma$ is the spin Hall angle. By fitting the amplitude of the Hanle curve with respect to $L$ with Eq. (1) (see Fig. 2b), the detected non-local signal indeed seems consistent with spin-charge interconversion and with previous experiments ${ }^{15}$. Here, by fitting to Eq. (1) we extract a spin diffusion length of $\lambda_{s}$ $=863 \pm 56 \mathrm{~nm}$ and a spin polarization of $\gamma=12.9 \pm 0.6 \%$, in good agreement with previous results ${ }^{15}$.

The $B$-field dependence of the resistance reduction due to the Hanle effect is given by ${ }^{23}$ :

$$
R_{N L}(B)=\frac{w}{2} \gamma^{2} R_{S} \operatorname{Re}\left(\left(\frac{\sqrt{1+i \tau_{s} \Gamma B}}{\lambda_{s}}\right) e^{-\frac{L \sqrt{1+i \tau_{s} \Gamma B}}{\lambda_{s}}}\right)
$$

where $\Gamma$ is the electron gyromagnetic ratio, $\Gamma=1.76 \times 10^{11} \mathrm{rad} /(\mathrm{s} \cdot T)$, and $\tau_{s}$ is the spinrelaxation time of carriers in the 2DEG. The dotted lines in Fig. 2a corresponds to the best fits of the data with Eqs. (1-2). From these fits, we extract the values of $\lambda_{s}, \gamma$ and $\tau_{s}$ shown in the table of Fig. 2c. Overall, we obtain values for $\lambda_{s}$ and $\gamma$ are very consistent with those extracted from the fit of $\Delta R_{N L}(L)$ by Eq. (1), and a spin-relaxation time of $\tau_{s} \approx 2$ ps.

a

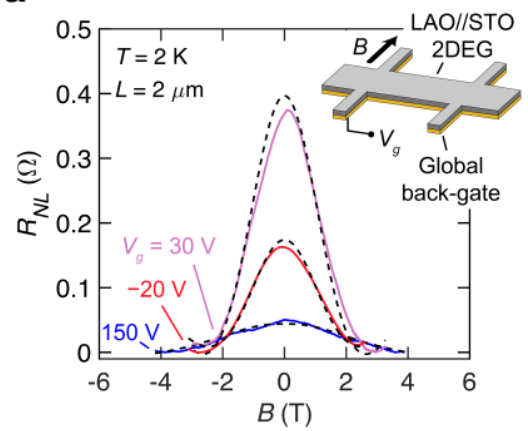

d

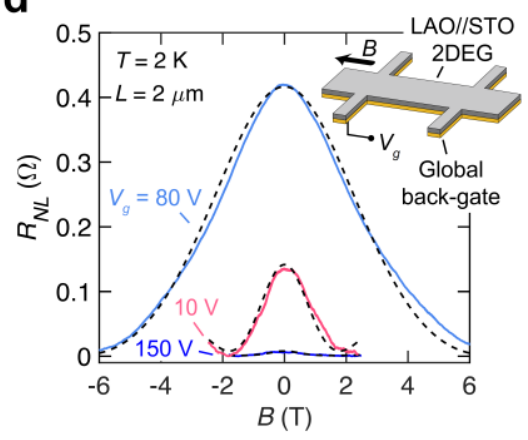

b

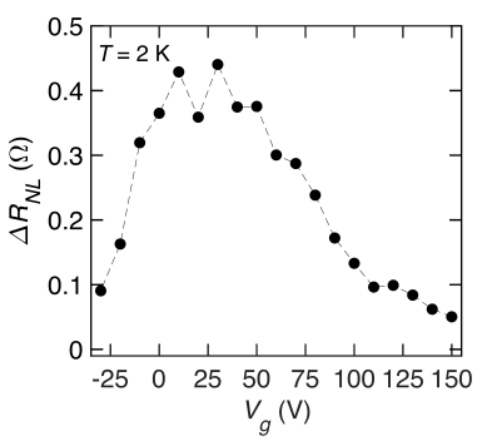

e

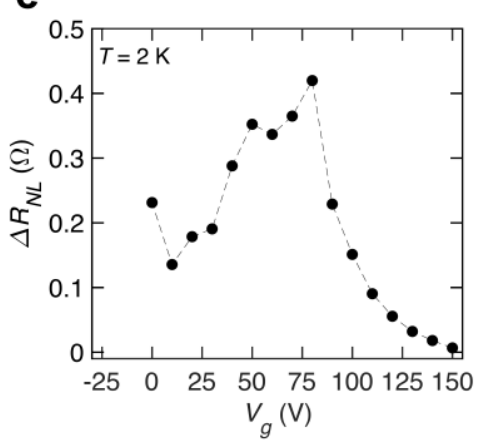

C

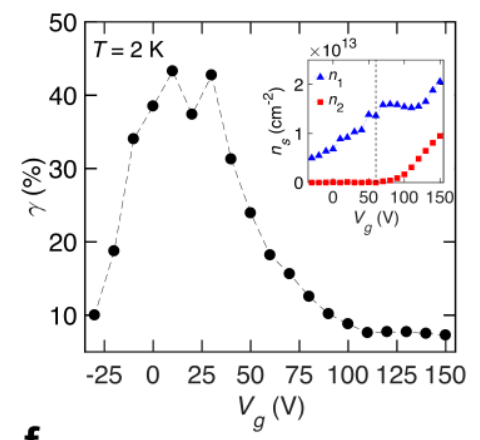

f

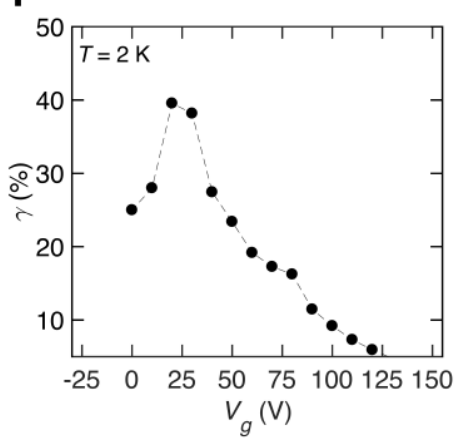

Figure 3. $a$, Non-local resistance, $R_{N L}$ as a function of the magnetic field applied along $x, B$, for three representative gate voltages at $2 K$, for a probe spacing of $2 \mu \mathrm{m}$. $\boldsymbol{b}$, Non-local spin 
resistance amplitude, $\Delta R_{N L}$ as a function of the gate voltage, $V_{g} . c$, Spin Hall angle, $\gamma$, extracted by fits of the data to Eqs (1-2). The inset of $c$ shows the individual sub-band carrier densities $\left(n_{1}, n_{2}\right)$ deduced from Hall measurements as a function of $V_{g}$, which positions the Lifshitz transition (one to two sub-band occupation) at $V_{g} \approx+50 \mathrm{~V} . d, e, f$, same as in $a, b, c$ except with B applied along $y$.

We then performed Hanle experiments as a function of the back gate voltage $V_{g}$, to probe its influence on the coupled charge-spin and spin-charge conversion processes. Representative results for three gate voltages are displayed in Fig. $3 a$, and the gate dependence of $\Delta R_{N L}$ is plotted in Fig. 3b. $\Delta R_{N L}$ shows a highly non-monotonic dependence with $V_{g}$, exhibiting a maximum at $V_{g} \approx+10-40 \mathrm{~V}$. By fitting the non-local resistance data simultaneously to Eqs. (1-2), we extract $\lambda_{s}, \tau_{s}$ (see Supporting Figure S2) and $\gamma$, plotted in Fig. 3c. The non-monotonic dependence of $\Delta R_{N L}$ with the back-gate voltage appears to originate from a large modulation of $\gamma$ between $5-40 \%$, with a maximum at $V_{g} \approx+10-40 \mathrm{~V}$. Remarkably, this maximum practically coincides with the Lifshitz transition ${ }^{25}$ occurring at $V_{g} \approx+50 \mathrm{~V}$ for $n_{s}$ $\approx 1.3 \times 10^{13} \mathrm{~cm}^{-2}$, as deduced from the analysis of the Hall resistance (see Supporting Figure S1), and corresponding to the onset of the occupancy of the first heavy $d_{x z / y z}$ sub-band (with carrier density $n_{2}$ in the inset of Fig. $3 c$ ). $\lambda_{s}$ and $\tau_{s}$ both show an overall positive correlation with $V_{g}$ (see Supporting Figure S2), reaching their largest values for the largest $V_{g}$. This increase is consistent with the onset of population of the $d_{x z / y z}$ sub-bands, which are known to result in higher electron mobilities ${ }^{26}$. Furthermore, the spin-diffusion length and the electron mobility display a strong correlation (see Supporting Information), as expected from the standard driftdiffusion equation within the Elliot-Yafet mechanism of spin-relaxation.

While these data indicate a strong spin-charge interconversion efficiency, highly tunable by an electric field, they cannot discriminate between EE and 2D-SHE as the underlying mechanism, since in both cases the applied magnetic field (along $x$ ) is perpendicular to the expected spin direction (along y for EE and along $z$ for 2D-SHE). To address this issue, we have performed similar experiments with the field applied along $y$, see Fig. $3 d-f$. The shape of the obtained Hanle curves is comparable to those obtained with the field along $x$, which indicates that the precessing spins are initially perpendicular to both $x$ and $y$, i.e. oriented along $z$ and identifies the 2D-SHE as the dominant mechanism for charge-spin interconversion. This is 
confirmed by measurements with the field applied along z (see Supporting Figure S3)) that show an inverted Hanle shape, expected when the spins have an initial average orientation parallel to the external magnetic field, in the presence of spin-randomizing processes.

The gate dependence of the Hanle signal with the field along y is shown in Fig. 3d-e, and bears a strong resemblance to that measured with the field along $x$, with a maximum for $V_{g} \approx 50-75 \mathrm{~V}$. We fitted independently the Hanle curves for this measurement geometry using Eqs. (1-2) and the extracted $\gamma$. Its gate dependence (Fig. 3f) shows a maximum value of about $40 \%$ at $V_{g} \approx 25-35 \mathrm{~V}$, and strongly resembles its counterpart with $B$ along $\mathrm{x}$. This further confirms that the 2D spin Hall effect is the main mechanism at play, and that $\gamma$ can be identified as a spin Hall angle. Its value, amounting to a maximum of about $45 \%$, is among the highest reported for oxide materials ${ }^{27,28}$ and larger than typical values found in heavy metals.

a

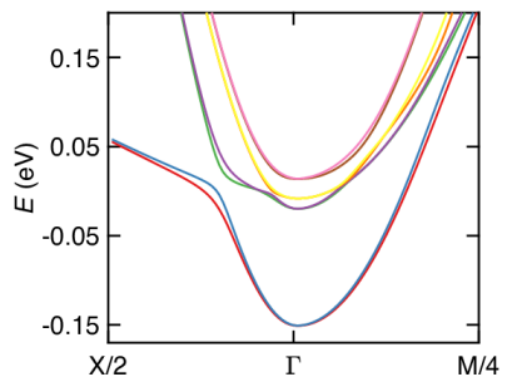

b

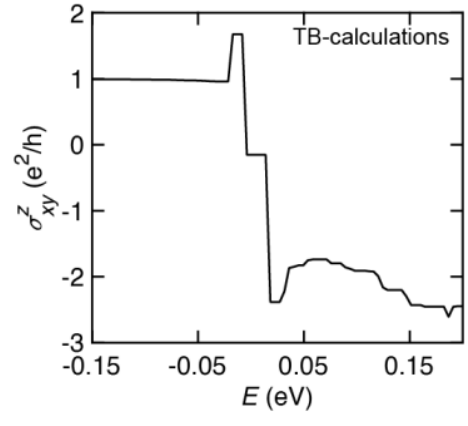

C

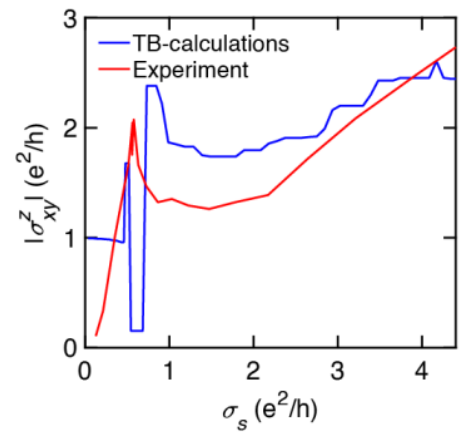

Figure 4. a, Calculated energy dispersion of the spin-split sub-bands along high symmetry crystal directions. $\boldsymbol{b}$, Calculated spin Hall conductance, $\sigma_{x y}^{z}$, as a function of the energy, $E . \boldsymbol{c}$, absolute value of $\sigma_{x y}^{Z}$ calculated and obtained experimentally from $\gamma$ in Fig. 3c, as a function of the sheet conductance, $\sigma_{s}$. The experimentally observed maxima of $\gamma$ is reasonably well captured by the calculations.

To further understand the nature of this large spin Hall angle ${ }^{29}$ and pinpoint the $2 \mathrm{D}$ spin Hall effect as the origin of the spin current generation, we have performed a theoretical modelling of LAO//STO (see Methods and Supporting Section S1) ${ }^{23}$. We theoretically determine the spin Hall conductance of the 2DEG, given as the product of the effective spin Hall angle by the sheet conductance $\sigma_{x y}=\gamma \sigma_{s}$, using a tight-binding (TB) form and compute the eigenenergies and eigenvectors for each wavevector $k$ in the Brillouin Zone (BZ) relevant to the electronic bands of LAO//STO (see Fig. $4 a)^{30}$. To quantitatively capture the Edelstein effect 
observed in these STO-based heterostructures, we have furthermore included spin-orbital terms in the TB-calculations. Using the Kubo-Streda formalism in the clean limit we have thus obtained the intrinsic spin Hall conductance of the 2DEG as $\sigma_{x y}(\mu)=$ $e \hbar \sum_{n} \int_{B Z} \Omega_{n}^{Z}(k) f_{n}\left(\epsilon_{k}^{n}-\mu\right) d^{2} k$, where we have summed the spin curvature, $\Omega_{n}^{Z}$, over filled states and all $k$ in the BZ. $f_{n}$ is the Fermi function for the band with index $n$. Subsequently, we computed the evolution of $\sigma_{x y}$ with respect to the chemical potential, $\mu$, (i.e. the energy, $E$, see Fig $4 \mathrm{~b})$. Here we note that the quantized plateaus in $\sigma_{x y}$ present at lower values of the chemical potential correspond to the occupation of consecutive $d_{x y}$ bands and are typical of a Rashba effect ${ }^{12}$. The fluctuations that are seen in $\sigma_{x y}$ at higher energy are associated with the Lifshitz transition ${ }^{25}$ and the filling of $d_{x z / y z}$ states where the system becomes strongly multiorbital with a complex intertwining of the bands ${ }^{31}$.

In order to directly compare our experimental results with the calculations, we relate the chemical potential and the sheet conductance from the TB model, and plot the spin Hall conductance as a function of the sheet conductance (see Supporting Fig. 1) for both the experimental results and the TB calculations (see Fig. 4c). It is evident from Fig. 4c that both the presence and magnitude of the non-monotonic behaviour of the spin Hall angle observed experimentally is reasonably well captured by the TB calculations, qualitatively and quantitatively. Based on this agreement, we see fit to conclude that the 2D spin Hall effect is indeed the origin of the generated spin current in our LAO//STO Rashba nanodevices.

Theoretically, the 2D-SHE is well described via the linear response theory and Kubo's formula giving the transverse conductance $\sigma_{x y}^{z}=\sigma_{x y}^{\Sigma}+\sigma_{x y}^{\mathrm{C}}$, that comprises two-parts: a bare (source) term, $\sigma_{x y}^{\Sigma}$, little dependent on the spin-flip processes, and a second contribution given by the so-called vertex corrections, $\sigma_{x y}^{\mathrm{C}}$ (see Supporting Section S2). The latter expresses the role of the spin-diffusion processes on the spin-accumulation appearing via IEE mechanisms and initially generated from $\sigma_{x y}^{\Sigma}$. However, it is generally proposed (see e.g. Ref. ${ }^{32}$ ) that in homogeneous Rashba 2DEGs, both terms exactly cancel each other in the case of klinear splitting departing from the exact LAO/STO case. However, our double crossbar geometry makes these devices essentially inhomogeneous, avoiding in fine the cancellation of the spin-Hall conductance $\sigma_{x y}^{z}$. This arises because the devices are patterned into lateral spin-diffusive channels, thus introducing a strong spin-flip contribution to the equation dynamics linking spin-current and spin-accumulation trough the spin relaxation time $\tau_{s}$. It 
follows that the spin-accumulation profile throughout the lateral channel is strongly affected by $\tau_{s}$, resulting in a significant reduction of both the spin-accumulation responsible for IEE and vertex corrections (also at the region of the injected current). As claimed in Refs. ${ }^{33,34}$, we here conclude that due to spin-flip processes along the lateral direction $\left|\sigma_{x y}^{\mathrm{C}}\right|<\left|\sigma_{x y}^{\Sigma}\right|$, which results in a non-zero spin Hall conductance. A possible longer spin-relaxation time along the direction normal to the layer, as already experimentally evidenced in other types of confined Rashba system ${ }^{35}$ may enhance the characteristic spin-diffusion length and size of SHE-ISHE compared to IEE in-plane effects.

In summary, we have realized spin transport and demonstrated the electric-field control of spin-charge interconversion in nanodevices built from a single material system, the 2DEG that forms at the LAO//STO interface. The conversion process proceeds through the direct and inverse 2D spin Hall effects, and the spin Hall angle can be electrically tuned from 10 to over $40 \%$. Our results now call for additional experiments with local top gates ${ }^{36}$, in which one could selectively tune spin generation, transport and detection. More importantly, they highlight the potential of oxide interfaces for low-power spin-orbitronics ${ }^{19}$, beyond conventional materials systems.

\section{MATERIALS AND METHODS}

\section{Sample growth and device fabrication}

To obtain the Hall bar devices, a single unit cell epitaxial $\mathrm{LaAlO}_{3}$ (as confirmed from an in-situ RHEED oscillation) was first deposited using pulsed laser deposition (PLD) on a $\mathrm{TiO}_{2}-$ terminated $\mathrm{SrTiO}_{3}$ (STO) substrate $\left(10 \times 10 \times 0.5 \mathrm{~mm}^{3}\right.$, miscut angle $<0.1$ degree $)$ in an oxygen atmosphere of $2 \times 10^{-4} \mathrm{mbar}$ at $730^{\circ} \mathrm{C}$. The LAO was ablated from a crystalline LAO target, with a target-substrate distance of $\sim 5.5 \mathrm{~cm}$, by a $\operatorname{KrF}$ laser $(\lambda=248 \mathrm{~nm})$ with a repetition rate of 1 $\mathrm{Hz}$ and laser fluence of $1 \mathrm{~J} / \mathrm{cm}^{2}$. After the LAO deposition, the sample was cooled to $T=550{ }^{\circ} \mathrm{C}$ at a pressure of $p_{O 2}=0.1 \mathrm{mbar}$. Once at $T=550{ }^{\circ} \mathrm{C}$, the sample was annealed $p_{O 2}=200 \mathrm{mbar}$ for one hour. Finally, the sample was brought to room temperature by passive cooling at $p_{O 2}$ = 200 mbar. Subsequently, the LAO(1u.c.)//STO sample was then prepared with e-beam PMMA A6 resist soft-mask according to the desired Hall bar design by e-beam lithography using standard exposure and development conditions. The sample was then returned to the 
PLD chamber for in-situ oxygen plasma cleaning ${ }^{21}$ prior to deposition of $30 \mathrm{~nm}$ amorphousLAO (a-LAO) at room temperature and an oxygen pressure $<1 \times 10^{-6}$ mbar. Following this deposition, the remaining PMMA resist was removed by lift-off in organic solvent leaving patterned regions with a-LAO(30nm)/LAO(1u.c.)//STO displaying interface conductivity (which for simplicity in the main text are referred to as LAO//STO). Note that these regions of interface conductivity are interspaced by insulating regions of LAO(1u.c.)//STO. Finally, the sample was mounted on a chip-carrier with a sample-wide back-gate of magnetron sputtered $\mathrm{Ti}(10 \mathrm{~nm}) / \mathrm{Au}(50 \mathrm{~nm})$.

\section{Transport measurements}

Low temperature electrical transport measurements were performed on the patterned Hall bar devices using a standard AC lock-in technique $\left(I_{A C}=20 \mathrm{nA}-2 \mu \mathrm{A}, f_{A C}=77.03 \mathrm{~Hz}\right)$ in a Quantum Design Dynacool cryostat at temperatures between $2 \mathrm{~K}$ and $300 \mathrm{~K}$ and magnetic fields between $-9 \mathrm{~T}$ and $9 \mathrm{~T}$. Prior to any back-gate voltage studies were performed, the LAO//STO devices were subjected to a so-called forming step ${ }^{37}$ at $2 \mathrm{~K}$, where the back-gate voltage were cycled several times $(>4)$ between $-100 \mathrm{~V}$ and $+150 \mathrm{~V}$ to ensure no irreversible changes would occur in the interface system upon application of the back-gate voltage in the actual experiment. Note that this low temperature forming step was repeated following all occasions the sample was brought above $105 \mathrm{~K}$. Moreover, at each new cooldown, the samples were always cooled with the back-gate electrostatically grounded.

\section{Tight binding calculations}

The tight-binding model (see Supplemental Section S1) allows us to compute the eigenvalues $\epsilon_{n}(k)$ and eigenfunctions $|n\rangle$ of the 2DEG at each $k$-point in the BZ. Using those, we determine the local spin curvature of the $n^{\text {th }}$ band at wavevector $\mathrm{k}$ :

$$
\Omega_{n}^{z}(k)=\sum_{m \neq n} \frac{\left\langle n\left|\hat{v}_{x}^{z}(k)\right| m\right\rangle\left\langle m\left|\hat{v}_{y}(k)\right| n\right\rangle-(x \leftrightarrow y)}{\left(\epsilon_{n}(k)-\epsilon_{m}(k)\right)^{2}}
$$

Where $\hat{v}_{\alpha}=\frac{1}{\hbar} \partial_{k_{\alpha}} \mathcal{H}$ and $\hat{v}_{\alpha}^{Z}=\frac{\hbar}{2}\left\{\hat{v}_{\alpha}, \hat{\sigma}_{z}\right\}$ are the current and spin current operators, respectively. 
We then derive the spin Hall conductivity $\sigma_{x y}$ for each value of the chemical potential following the protocol outlined in the main text. Hall measurements give the change in subband population with respect to the gate voltage. Furthermore, the tight-binding band structure of the material allows us to compute the evolution of the charge filling with respect to the chemical potential. We use the Lifshitz transition as a reference point and assume that the population ratio of the heavy to the light sub-band is the same for both the experimental and numerical values. This yields a correspondence between the chemical potential $\mu$ and $V_{g}$ and hence between $\mu$ and the sheet conductance $\sigma_{s}$.

\section{ASSOCIATED CONTENT}

Supporting Information is available for this paper at [insert link for SI here].

\section{AUTHOR INFORMATION}

\section{Corresponding Author}

*e-mail: manuel.bibes@cnrs-thales.fr. All correspondence should be addressed to M.B.

\section{Author Contributions}

$M B$ and LV designed the experiment and MB supervised the study. FT prepared the samples with the help of DCV. FT performed the transport experiments and analysed them with the help of $M B, H J, A B, A F, L V, J P A$ and $P N$. PB and MG performed tight binding and transport calculations. FT and MB wrote the paper with inputs from all authors.

\section{Funding Sources}

F. Trier acknowledges support by research grant VKR023371 (SPINOX) from VILLUM FONDEN. This work received support from the ERC Consolidator grant no. 615759 "MINT", the French Research Agency (ANR) as part of the "Investissement d'Avenir" program (LABEX NanoSaclay, ref ANR-10-LABX-0035) through project "AXION" and ANR program OISO (ANR-17-CE24-0026- 
03), TOPRISE (ANR-16-CE24-0017) and the Laboratoire d'Excellence LANEF (ANR-10-LABX-5101).

\section{ACKNOWLEDGMENT}

The authors thank the CTU Minerve Nanotechnology platform where electron beam lithography was performed and B. van Wees for useful comments.

\section{REFERENCES}

1. Santander-Syro, A. F. et al. Two-dimensional electron gas with universal subbands at the surface of SrTiO3. Nature 469, 189-193 (2011).

2. Ohtomo, A. \& Hwang, H. Y. A high-mobility electron gas at the LaAlO3/SrTiO3 heterointerface. Nature 427, 423-426 (2004).

3. Chen, Y. et al. Metallic and Insulating Interfaces of Amorphous $\mathrm{SrTiO}_{3}$-Based Oxide Heterostructures. Nano Lett. 11, 3774-3778 (2011).

4. Rödel, T. C. et al. Universal Fabrication of 2D Electron Systems in Functional Oxides. Adv. Mater. 28, 1976-1980 (2016).

5. Chen, Y. Z. et al. A high-mobility two-dimensional electron gas at the spinel/perovskite

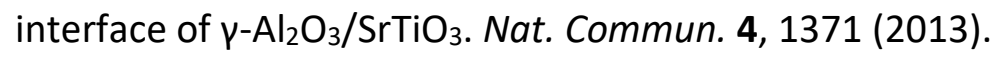

6. Reyren, N. et al. Superconducting Interfaces Between Insulating Oxides. Science 317, 1196-1199 (2007).

7. Bychkov, Y. A. \& Rashba, E. I. Properties of a 2 D electron gas with lifted spectral degeneracy. JETP Lett. 39, 78-81 (1984).

8. Caviglia, A. D. et al. Electric field control of the LaAlO3/SrTiO3 interface ground state. Nature 456, 624-627 (2008). 
9. Caviglia, A. D. et al. Tunable Rashba Spin-Orbit Interaction at Oxide Interfaces. Phys. Rev. Lett. 104, 126803 (2010).

10. Edelstein, V. M. Spin polarization of conduction electrons induced by electric current in two-dimensional asymmetric electron systems. Solid State Commun. 73, 233-235 (1990).

11. Sánchez, J. C. R. et al. Spin-to-charge conversion using Rashba coupling at the interface between non-magnetic materials. Nat. Commun. 4, (2013).

12. Sinova, J. et al. Universal Intrinsic Spin Hall Effect. Phys. Rev. Lett. 92, 126603 (2004).

13. Lesne, E. et al. Highly efficient and tunable spin-to-charge conversion through Rashba coupling at oxide interfaces. Nat. Mater. 15, 1261-1266 (2016).

14. Wang, Y. et al. Room-Temperature Giant Charge-to-Spin Conversion at the $\mathrm{SrTiO}_{3}-$ $\mathrm{LaAlO}_{3}$ Oxide Interface. Nano Lett. 17, 7659-7664 (2017).

15. Jin, M.-J. et al. Nonlocal Spin Diffusion Driven by Giant Spin Hall Effect at Oxide Heterointerfaces. Nano Lett. 17, 36-43 (2017).

16. Chauleau, J.-Y. et al. Efficient spin-to-charge conversion in the $2 \mathrm{D}$ electron liquid at the LAO/STO interface. EPL Europhys. Lett. 116, 17006 (2016).

17. Vaz, D. C. et al. Mapping spin-charge conversion to the band structure in a topological oxide two-dimensional electron gas. Nat. Mater. 18, 1187 (2019).

18. Shanavas, K. V., Popović, Z. S. \& Satpathy, S. Theoretical model for Rashba spin-orbit interaction in d electrons. Phys. Rev. B 90, 165108 (2014).

19. Manipatruni, S. et al. Scalable energy-efficient magnetoelectric spin-orbit logic. Nature 565, 35-42 (2019).

20. Varignon, J., Vila, L., Barthélémy, A. \& Bibes, M. A new spin for oxide interfaces. Nat. Phys. 14, 322-325 (2018). 
21. Bjørlig, A. V. et al. Nanoscale patterning of electronic devices at the amorphous LaAlO 3 /SrTiO ${ }_{3}$ oxide interface using an electron sensitive polymer mask. Appl. Phys. Lett. 112, 171606 (2018).

22. Herranz, G. et al. High Mobility in $\mathrm{LaAlO}_{3} / \mathrm{SrTiO}_{3}$ Heterostructures: Origin, Dimensionality, and Perspectives. Phys. Rev. Lett. 98, 216803 (2007).

23. Abanin, D. A., Shytov, A. V., Levitov, L. S. \& Halperin, B. I. Nonlocal charge transport mediated by spin diffusion in the spin Hall effect regime. Phys. Rev. B 79, 035304 (2009).

24. Reyren, N. et al. Gate-Controlled Spin Injection at LaAlO 3 / SrTiO 3 Interfaces. Phys. Rev. Lett. 108, 186802 (2012).

25. Joshua, A., Pecker, S., Ruhman, J., Altman, E. \& Ilani, S. A universal critical density underlying the physics of electrons at the LaAlO3/SrTiO3 interface. Nat. Commun. 3, 1129 (2012).

26. Bell, C. et al. Dominant Mobility Modulation by the Electric Field Effect at the LaAlO 3 / SrTiO 3 Interface. Phys. Rev. Lett. 103, 226802 (2009).

27. Everhardt, A. S. et al. Tunable charge to spin conversion in strontium iridate thin films. Phys. Rev. Mater. 3, 051201 (2019).

28. Vaz, D. C., Barthélémy, A. \& Bibes, M. Oxide spin-orbitronics: New routes towards lowpower electrical control of magnetization in oxide heterostructures. Jpn. J. Appl. Phys. 57, 0902A4 (2018).

29. Tanaka, T. et al. Intrinsic spin Hall effect and orbital Hall effect in $4 \mathrm{~d}$ and $5 \mathrm{~d}$ transition metals. Phys. Rev. B 77, 165117 (2008).

30. Cancellieri, C. et al. Polaronic metal state at the LaAlO3/SrTiO3 interface. Nat. Commun. 7, 10386 (2016). 
31. Vivek, M., Goerbig, M. O. \& Gabay, M. Topological states at the (001) surface of SrTiO 3. Phys. Rev. B 95, (2017).

32. Dimitrova, O. V. Spin-Hall conductivity in a two-dimensional Rashba electron gas. Phys. Rev. B 71, 245327 (2005).

33. Seibold, G., Caprara, S., Grilli, M. \& Raimondi, R. Intrinsic spin Hall effect in systems with striped spin-orbit coupling. EPL Europhys. Lett. 112, 17004 (2015).

34. Kato, T., Ishikawa, Y., Itoh, H. \& Inoue, J. Anomalous Hall effect in spin-polarized twodimensional electron gases with Rashba spin-orbit interaction. New J. Phys. 9, 350-350 (2007).

35. Ghiasi, T. S., Kaverzin, A. A., Blah, P. J. \& van Wees, B. J. Charge-to-Spin Conversion by the Rashba-Edelstein Effect in Two Dimensional van der Waals Heterostructures up to Room Temperature. Nano Lett. 19, 5959-8966 (2019).

36. Hurand, S. et al. Field-effect control of superconductivity and Rashba spin-orbit coupling in top-gated LaAlO3/SrTiO3 devices. Sci. Rep. 5, 12751 (2015).

37. Biscaras, J. et al. Limit of the electrostatic doping in two-dimensional electron gases of $\mathrm{LaXO}_{3}(\mathrm{X}=\mathrm{Al}, \mathrm{Ti}) / \mathrm{SrTiO}_{3}$. Sci. Rep. 4, 6788 (2014). 